core, in which case the output of radiation per unit mass of star would correspondingly vary. The view expressed by Russell and Jeans, that differences in this quantity depend on differences in amount of a hypothetical active material, is thus in accord with the hypothesis.

The question of reconciling it-or otherwise-with current views of the course of stellar evolution, is another matter which, if further consideration appears to warrant it, may be dealt with in a future communication. At present I remark only that it suggests an origin for stars in an assemblage of protons and high-speed electrons - the problem of whether and how these have a beginning lies deeper-and an unforced explanation for the occurrence of 'white dwarfs,' differing entirely from either of those proposed by Dr. Jeans or Prof. Eddington.

Department of Physics,

University of Adelaide, July 5.

Kerr Grant.

The Volatility and Dissociation of Borax.

Kolthoff ( $J$. Amer. Chem. Soc., I926, 48, I447) states that he has been unable to confirm our statement (Jour. Chem. Soc., I925, r27, I50) that fused borax loses sodium oxide. He reports that " even after the substance had been heated for two hours at $800^{\circ}$ the weight did not change." His experiments, however, appear to be scarcely precise enough to prove that borax on heating (I) is not volatile, and (2) does not change in composition; as a matter of fact the volatility of borax at high temperatures is well established, having been observed by HoskynsAbrahall (Jour. Chem. Soc., I892, 6I, 650), Leonard (Chem. News, r898, 77, IO4), and Smith and Van Haagen ("The Atomic Weights of Boron and Fluorine," Carnegie Inst. Washington, Publication No. 267, I9I8).

We would direct attention especially to the lastmentioned publication, where it is stated that "it is certain and not at all surprising that borax cannot be fused for any considerable time without loss." This evidence is so conclusive as to require no emphasis here. There is, however, another interesting piece of evidence. The inside of the silica muffle used for the fusions of borax in our investigation was completely coated with a white opaque enamel, about 0.06 inch thick, of a product of a reaction between the volatilised material and the silica. Clearly the salt had volatilised in some quantity, and with such a volatile substance, selective loss of the constituents could be detected only by analysing the residues. We believe that the analyses given in our paper can only be interpreted to mean that borax on prolonged fusion leaves a residue poorer in sodium oxide than is required by the formula $\mathrm{Na}_{2} \mathrm{~B}_{4} \mathrm{O}_{3}$, and afford definite evidence of a preferential loss of sodium
oxide. P. L. RoBINSON.

University of Durham, Armstrong College, Newcastle-on-Tyne, August Io.

\section{Photographic Theory.}

IN the course of photographic investigations at the Royal Observatory, Edinburgh, it has been found by Mr. E. A. Baker that the initial stages of the photographic action, including the deviations from the reciprocity law, are calculable and well represented by assuming that the developable product is formed in two stages, each requiring one quantum; and that the product of the first stage returns in the absence of further stimulus to its original sensitive state, according to the usual law governing the progress of a mono-molecular change. In the case of process plates, where the grains are small and nearly uniform in size, the experimental results agree so closely with those deduced on this theory as to warrant the belief that the departures from it with fast emulsions, where not due to the reversal action, are to be accounted for by the diversity of grain size. The results of these investigations will be submitted to the Royal Society of Edinburgh during the coming session.

Experimental results for single layer fast emulsions and weak light are much to be desired in this connexion. The purpose of the present note is to express the hope that some of the researches at present in progress at different places may be directed to that end, and that results already obtained may be published.

Royal Observatory, Edinburgh, August $\mathrm{r}_{3}$.

\section{Kaufmann's Experiment and the Spinning Electron.}

In Nature of August 2x Dr. L. C. Jackson quotes Wentzel as having supposed a force

$$
(\mu[v X]) / c \text {. }
$$

to act on a magnetic electron with moment $\mu$ moving with velocity $v$ in electric field $X(c$ is the velocity of light). He deduces from (I) that Kaufmann's experiment shows that the electron cannot have a magnetic moment as large as a Bohr magneton.

Wentzel, however (Zeitschrift für Physik, 37, p. 9Ir), used (I) as Storüngsfunktion, i.e. energy. (For an electron describing a periodic orbit in an atom energy

$$
(\mu[\because X]) / 2 c . . \quad . \quad . \quad . \quad \cdot(2)
$$

would, I think, lead to the correct first order perturbation, but the equations of motion are not of Hamiltonian form and (2) would not apply in general.) Thus Dr. Jackson's argument rests on a mistake. His formula can be seen to be wrong dimensionally. ' $H e$ ' has not the same dimensions as ' $X \mu / c$.' In fact, extra force on a magnetic electron will depend on the gradient of the field. Kaufmann's experiment in no way precludes the electron from having a Bohr magneton of magnetic moment. L. H. THomas.

Trinity College, Cambridge.

\section{Liver Extracts in the Treatment of Malignant Disease.}

The letter from Dr. J. R. Howitt in NAture of August 2I, p. 263, appears to be based upon an extraordinary presumption. The liver, in early fotal life, is large because it is an active blood-forming organ, but Dr. Howitt seems to suppose that it must have some endocrine function relating to growth. If this idea were correct, it would seem rational to presume that it was a growth-accelerating hormone, for the embryo is growing rapidly. Dr. Howitt seems to think that it is a growth-retarding or, at least, a growth-regulating hormone. That the method advocated may have clinical value is, of course, possible, but it is difficult to understand the theoretical basis of the treatment. I have no knowledge of decreased activity of growth in tumours when associated with enlargement of the liver due either to simple hypertrophy or to resumption of its blood-forming activity.

Institute of Pathology, A. Piney.

Charing Cross Hospital, London, W.C.2, August 22.

NO. 2967 , VOL. I I 8] 